\title{
Abrupt worsening of occult IgA nephropathy after the first dose of SARS-CoV-2 vaccination
}

\author{
Yoko Fujita $^{1} \cdot$ Keisuke Yoshida $^{1} \cdot$ Daisuke Ichikawa $^{1} \cdot$ Yugo Shibagaki $^{1} \cdot$ Masahiko Yazawa $^{1}$ (I)
}

Received: 24 September 2021 / Accepted: 27 November 2021 / Published online: 6 January 2022

(C) Japanese Society of Nephrology 2021

\begin{abstract}
Here, we report a case of abrupt onset of gross hematuria and nephrotic range proteinuria after the first dose of severe acute respiratory syndrome coronavirus 2 (SARS-CoV-2) vaccination, which led to a diagnosis of immunoglobulin A nephropathy (IgAN). A Japanese woman in their forties with a significant medical history of occult blood by urine dipstick test (over the past 3 years) presented with fever, chills, shivering, marked thrombocytopenia, and gross hematuria 9 days after the first dose of the BNT162b2 mRNA vaccine (Pfizer) against SARS-CoV-2 infection. Although thrombotic microangiopathy (TMA) was first suspected as the cause of the severe thrombocytopenia, TMA was clinically excluded after two sessions of plasma exchange were performed. Renal biopsy was performed as the patient's platelet count improved. We made a diagnosis of acute worsening IgAN, triggered by the first dose of SARS-CoV-2 vaccination. In this case, we speculated that vaccineinduced immune activation may be involved in the exacerbation of occult IgAN, leading to the definite diagnosis. We should pay more attention to the development/worsening of clinically significant kidney disease after SARS-CoV-2 vaccination not only in those with known glomerular disease but also in those with only mild urinary abnormality.
\end{abstract}

Keywords SARS-CoV-2 $\cdot$ Immunoglobulin A nephropathy $\cdot$ BNT162b2 mRNA vaccine

\section{Introduction}

During the severe acute respiratory syndrome coronavirus 2 (SARS-CoV-2) pandemic, mortality and morbidity increased in patients with kidney disease infected with SARS-CoV-2 [1]. Several reports have shown that apart from the coronavirus disease 2019 (COVID-19) infection itself, the SARSCoV-2 vaccination may be involved in the pathogenesis/

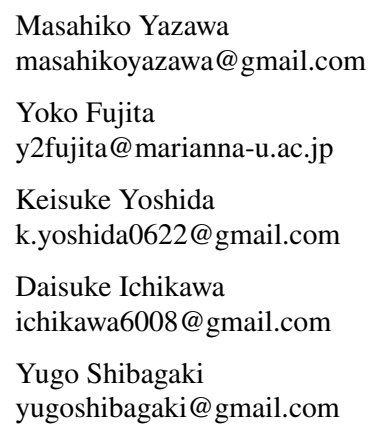

1 Division of Nephrology and Hypertension, Department of Internal Medicine, St. Marianna University School of Medicine, 2-16-1 Sugao, Miyamae-Ku, Kawasaki, Kanagawa 216-8511, Japan exacerbation of certain kidney diseases such as acute kidney disease, nephrotic syndrome, and glomerulonephritis [2]. Most reports on glomerulonephritis after vaccination are relapsing cases of immunoglobulin A nephropathy (IgAN), while only one case report describes a de novo case of IgAN [3-5], in which exacerbation of immune-mediated glomerular diseases by the vaccine could be the mechanism of action.

IgAN is the most common form of primary glomerulonephritis in the Asia-Pacific region, including Japan, and accounts for $20-40 \%$ of all glomerular diseases [6]. Due to the high prevalence of IgAN, there would be a huge number of patients with occult IgAN having only mild microscopic hematuria. We should pay more attention to patients with mild urinary abnormality after SARS-CoV-2 vaccination regardless of the pathologically diagnosed glomerulonephritis, especially IgAN.

Here, we report the case of a patient suspected to have occult IgAN with persistent urine occult blood over the past 3 years, who developed clinically and pathologically apparent IgAN 9 days after SARS-CoV-2 vaccination. 


\section{Case report}

A Japanese woman in her early forties with a significant history of urine occult blood [a few times of $(+1)$ to $(2+)$ ] and proteinuria $[(-)$ to $(1+)]$ by dipstick test over the past 3 years, without further workup presented with fever (body temperature, $38^{\circ} \mathrm{C}$ ), chills and shivering, back pain, general malaise, and gross hematuria 9 days after receiving the first dose of the BNTb162b2 SARS-CoV-2 vaccine (Pfizer). She had no history of previous infections such as tonsillitis or colitis. Polymerase chain reaction for SARSCoV-2 was negative. She was prescribed only acetaminophen for these symptoms.

Thirteen days after vaccination, the patient was referred and admitted to our hospital for hematuria, nephroticrange proteinuria, pyuria, and severe thrombocytopenia of $7.0 \times 10^{3} / \mu \mathrm{L}$. On admission, the fever had subsided, and obvious sore throat, purpura or petechiae, bloody stools, abdominal pain, or lower limb arthralgia were all absent. Table 1 and Fig. 1 show the clinical data on admission and clinical course after vaccination, respectively. On admission, urinalysis showed severe hematuria with a red blood cell count of 100/high power field (HPF), massive proteinuria of $18.13 \mathrm{~g} / \mathrm{gCr}$ (urine protein-to-creatinine ratio), and a mildly elevated serum creatinine level at $0.86 \mathrm{mg} /$ $\mathrm{dL}$ (previous level of $0.76 \mathrm{mg} / \mathrm{dL}$ ), which suggested the development or worsening of occult glomerular disease. Although she had nephrotic range proteinuria, her serum albumin was persistently greater than $3.0 \mathrm{~g} / \mathrm{dL}$. We did not measure the value of albuminuria on admission. Furthermore, proteinuria spontaneously resolved to almost within the normal range without any specific treatment. Thus, such high-level proteinuria would be affected gross hematuria at 9 days after vacctination and consistent with non-glomerular proteinuria. Due to the mildly elevated inflammation marker and urine leukocyte count (5-9 cells/ HPF), ampicillin/sulbactam (ABPC/SBT) was administered for 10 days for probable pyelonephritis.

The platelet count further decreased to $4.0 \times 10^{3} / \mu \mathrm{L}$ on day 2 of admission (day 13 after vaccination). Although gross hematuria resolved on day 15 after vaccination, the microscopic hematuria and proteinuria persisted. The creatinine level increased to $1.23 \mathrm{mg} / \mathrm{dL}$, indicating progression of renal dysfunction. No fragmented red blood cells were observed, and the lactate dehydrogenase level was normal. However, because of the rapid thrombocytopenia, we considered that the patient might have thrombotic microangiopathy (TMA), especially thrombotic thrombocytopenic purpura (TTP). Two sessions of plasma exchange (PE) therapy were performed until TTP was ruled out. Other causes of thrombocytopenia, such as pseudothrombocytopenia, disseminated intravascular coagulation, and immune thrombocytopenic purpura, were less likely, as shown in Table 1. Since thrombocytopenia and thrombus formation have been reported after COVID19 vaccination [7], we tested for antiplatelet antibodies and platelet factor 4, which were negative. Magnetic resonance imaging was performed due to persistent headache till day 17 after vaccination. No thrombus or intracranial hematoma was found. Thrombocytopenia improved to within normal level during her clinical course (Fig. 1).

Since her renal function did not improve completely with persistent hematuria and proteinuria, percutaneous renal biopsy was performed on day 20 after vaccination when the platelet count had improved. The biopsy specimen revealed 33 glomeruli with mild proliferation of the mesangial and endocapillary cells, 2 fibrous crescents (Fig. 2A), and 3 fibrocellular crescents (Fig. 2B), suggesting a mixed acute and chronic phase of glomerular disease. Of the 33 glomeruli, 6\% were global glomerulosclerosis (GGS), and interstitial fibrosis/tubular atrophy (IF/TA) was not noticeable. Fluorescent immunostaining showed predominant IgA deposition in the mesangium (Fig. 2C), with concurrent IgG, IgM, and C3 deposition. Electron microscopy showed mildly elevated electron-dense deposits in the mesangium and very mild foot-process effacement (Fig. 2D). There were no features of infection-related or other autoimmune disease-related glomerulonephritis. Thus, we finally made a diagnosis of IgAN with the Oxford MEST-C Classification [8] as M1-E0-S0-T0-C1.

The patient was discharged after all conditions had resolved. Two months after discharge, the serum creatinine level and proteinuria decreased to almost normal levels, while the microscopic hematuria persisted. The urinalysis test result gradually improved with time. No specific treatment for IgAN such as steroids, fish oil, and renin-angiotensin system (RAS) blockers was administered because the clinical course spontaneously and gradually resolved.

\section{Discussion}

This is a case of occult IgAN that developed a significant clinical flare 9 days after the patient received the first dose of the BNT162b2 mRNA COVID-19 vaccine, leading to a kidney biopsy that led to the definitive diagnosis. In retrospect, we speculated that this case was occult IgAN rather than a de novo one since the patient had had urine occult blood over the past 3 years and the biopsy findings were likely to show chronic rather than acute features. This report highlights that COVID-19 vaccination can exacerbate pre-existing occult and even un-diagnosed $\operatorname{IgAN}$ with significant clinical flares as urinary abnormalities and renal dysfunction. 
Table 1 Clinical data on admission

\begin{tabular}{|c|c|}
\hline & Day 12 (day of admission) \\
\hline \multicolumn{2}{|l|}{ Laboratory data } \\
\hline White blood cells $\left(10^{3} / \mu \mathrm{L}\right)$ & 7.5 \\
\hline Hemoglobin (g/dL) & 12.9 \\
\hline Platelet $\left(10^{3} / \mu \mathrm{L}\right)$ & 7.0 \\
\hline $\mathrm{CRP}(\mathrm{mg} / \mathrm{dL})$ & 1.67 \\
\hline Total bilirubin (mg/dL) & 0.5 \\
\hline AST (IU/L) & 21 \\
\hline ALT (IU/L) & 15 \\
\hline LDH (U/L) & 210 \\
\hline Total protein $(\mathrm{g} / \mathrm{dL})$ & 6.2 \\
\hline Albumin (g/dL) & 3.6 \\
\hline BUN (mg/dL) & 12.4 \\
\hline Creatinine (mg/dL) & 0.86 \\
\hline $\mathrm{eGFR}\left(\mathrm{mL} / \mathrm{min} / 1.73 \mathrm{~m}^{2}\right)$ & 57.5 \\
\hline $\mathrm{Na}(\mathrm{mEq} / \mathrm{L})$ & 140 \\
\hline $\mathrm{Cl}(\mathrm{mEq} / \mathrm{L})$ & 107 \\
\hline $\mathrm{K}(\mathrm{mEq} / \mathrm{L})$ & 3.8 \\
\hline PT-INR & 1.04 \\
\hline APTT (SEC) & 28.8 \\
\hline $\mathrm{D}$-dimer $(\mu \mathrm{g} / \mathrm{mL})$ & 2.0 \\
\hline IgA (mg/dL) (standard value: 93-393) & 155 \\
\hline C3 (mg/dL) (standard value: 65-135) & 88 \\
\hline C4 (mg/dL) (standard value: 13-35) & 18 \\
\hline Urine cast & Red blood cell $3+$ \\
\hline Proteinuria by dipstick test & $2+$ \\
\hline Hematuria by dipstick test & $3+$ \\
\hline Hematuria in urinary sediment (count/HPF) & $\begin{array}{c}>100 \text { (non-glomerular or } \\
\text { glomerular hematuria) }\end{array}$ \\
\hline White blood cells in urinary sediment (count/HPF) & $5 \backsim 9$ \\
\hline Urinary protein creatinine ratio $(\mathrm{g} / \mathrm{gCr})$ & 18.1 \\
\hline \multicolumn{2}{|l|}{ Thrombocytopenia associated laboratory results } \\
\hline Pseudo thrombocytopenia using EDTA & N/A \\
\hline Fragmented RBC & N/A \\
\hline ADAMS13 activity (IU/mL) (reference range: $0.75-1.5$ ) & 0.64 \\
\hline ADAMS13 antibody $(\mathrm{BU} / \mathrm{mL})($ reference range: $<0.5)$ & $<0.5$ \\
\hline STEC & Negative \\
\hline Direct coombs test & Negative \\
\hline Anti-nuclear antibody (standard value: $0-40$ ) & $<40$ \\
\hline Anti-platelet antibodies & Negative \\
\hline PF4 (ng/mL) (standard value: 0-20) & 16 \\
\hline PF4-heparin antibodies $(\mathrm{U} / \mathrm{mL})$ (standard value: $<1.0)$ & $<0.6$ \\
\hline Measles IgM (standard value: $0-0.8$ ) & 0.14 \\
\hline VZV IgM (standard value: $<0.8$ ) & 0.39 \\
\hline Rubella IgM (standard value: $0-0.8$ ) & 0.39 \\
\hline CMV antigen $(\mathrm{C} 10 / \mathrm{C} 11)$ & N/A \\
\hline CMV IgM (standard value: $0-0.8$ ) & 0.45 \\
\hline Mumps IgM (standard value: $0-0.8$ ) & 0.11 \\
\hline HSV IgM (standard value: $0-0.8$ ) & 0.22 \\
\hline EBV anti-VCA IgM (standard value: $0-0.5$ ) & 0.0 \\
\hline HIV antibody & Negative \\
\hline
\end{tabular}

$C R P$ C-reactive protein, $A S T$ aspartate aminotransferase, $A L T$ alanine aminotransferase, $L D H$ lactate dehydrogenase, $B U N$ blood urea nitrogen, $e G F R$ estimated glomerular filtration, $\mathrm{Na}$ serum sodium, $\mathrm{Cl}$ serum chloride, $K$ potassium, $P T$ prothrombin time-international normalized ratio, $A P T T$ activated partial thromboplastin time, $H P F$ high power field, $C r$ creatinine, EDTA ethylenediaminetetraacetic acid, $R B C$ red blood cell, ADAMTS13 a disintegrin-like and metalloproteinase with thrombospondin type 1 motifs $13, B U$ 
Table 1 (continued)

Bethesda units, STEC Shiga-toxin-producing Escherichia coli, PF4 platelet factor 4, VZV varicella-zoster virus, $C M V$ cytomegalovirus, $H S V$ herpes simplex virus, $E B V$ Epstein-Barr virus, $V C A$ virus capsid antigen, $H I V$ human immunodeficiency virus, N/A not available

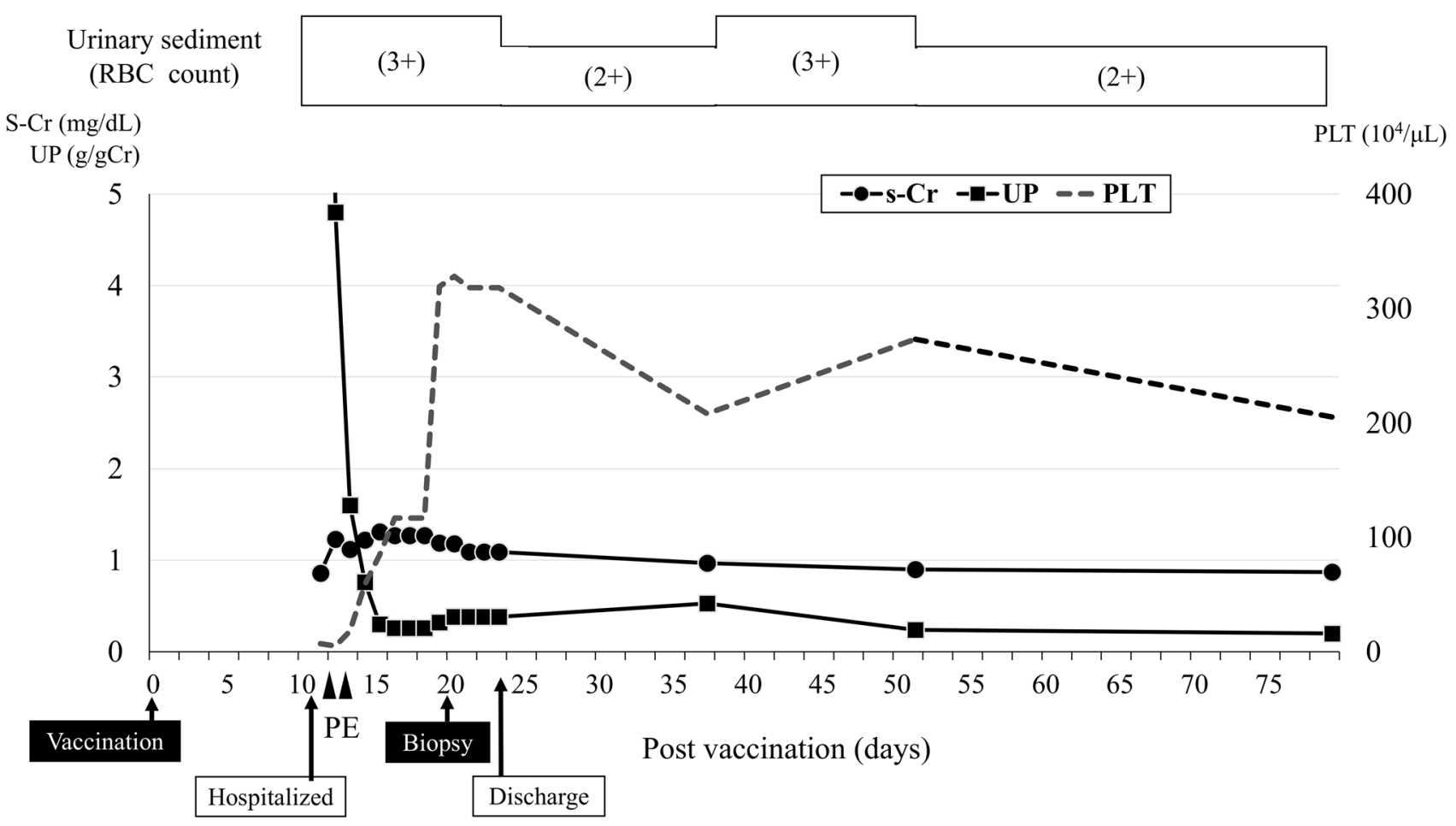

Fig. 1 Clinical course of key findings after vaccination. $S$ - $C r$ serum creatinine, $U P$ urinary protein, $R B C$ red blood cell, $P E$ plasma exchange, PLT platelet count

Relapsing or de novo cases of IgAN after vaccinations have been reported [3-5], in which cases showed gross hematuria immediately after the vaccinations but resolved spontaneously (Table 2). In our case, the abrupt development of gross hematuria following vaccination led to the definitive diagnosis of pre-existing occult IgAN. This is similar to case 4 illustrated in Table 2. However, the present case developed gross hematuria after the first dose of vaccination, and the onset of symptoms was later ( 9 days post first vaccination) than that of other cases (within $24 \mathrm{~h}$ after the second dose of vaccination). Although previous reports did not specify the degree of renal dysfunction or the amount of proteinuria before and after vaccination, this case showed elevated creatinine, nephrotic-range proteinuria, and gross hematuria. This suggests that the severity of the IgAN flare in our case was more significant than those mentioned in previous reports, which could also explain why the clinical improvement was slower (the trend of improvement continued even after 2 months of disease onset) than those in the previous reports described in Table 2.

We speculated that the exacerbation of the immune-mediated glomerular disease by the SARS-CoV-2 vaccine could be the mechanism of the exacerbation of IgAN. Vojdani et al. [9] hypothesized the existence of molecular mimicry between the spike protein of SARS-CoV-2 and autoantigens and pointed out that SARS-CoV-2 infection and the mRNA vaccine may lead to the development of immune-mediated diseases. In a small-scale experiment [10], it was reported that SARS-CoV-2 mRNA vaccination induced spike antigen-specific IgA and IgG. Both increased exponentially after vaccination and reached the peak in approximately 20 days. 
Fig. 2 Biopsy findings. A Periodic acid-Schiff stain $\times 200, \mathbf{B}$ periodic acid-Schiff stain $\times 200$, C immunofluorescence study $\times 200$, and $\mathbf{D}$ electron micrography $\times 4000$
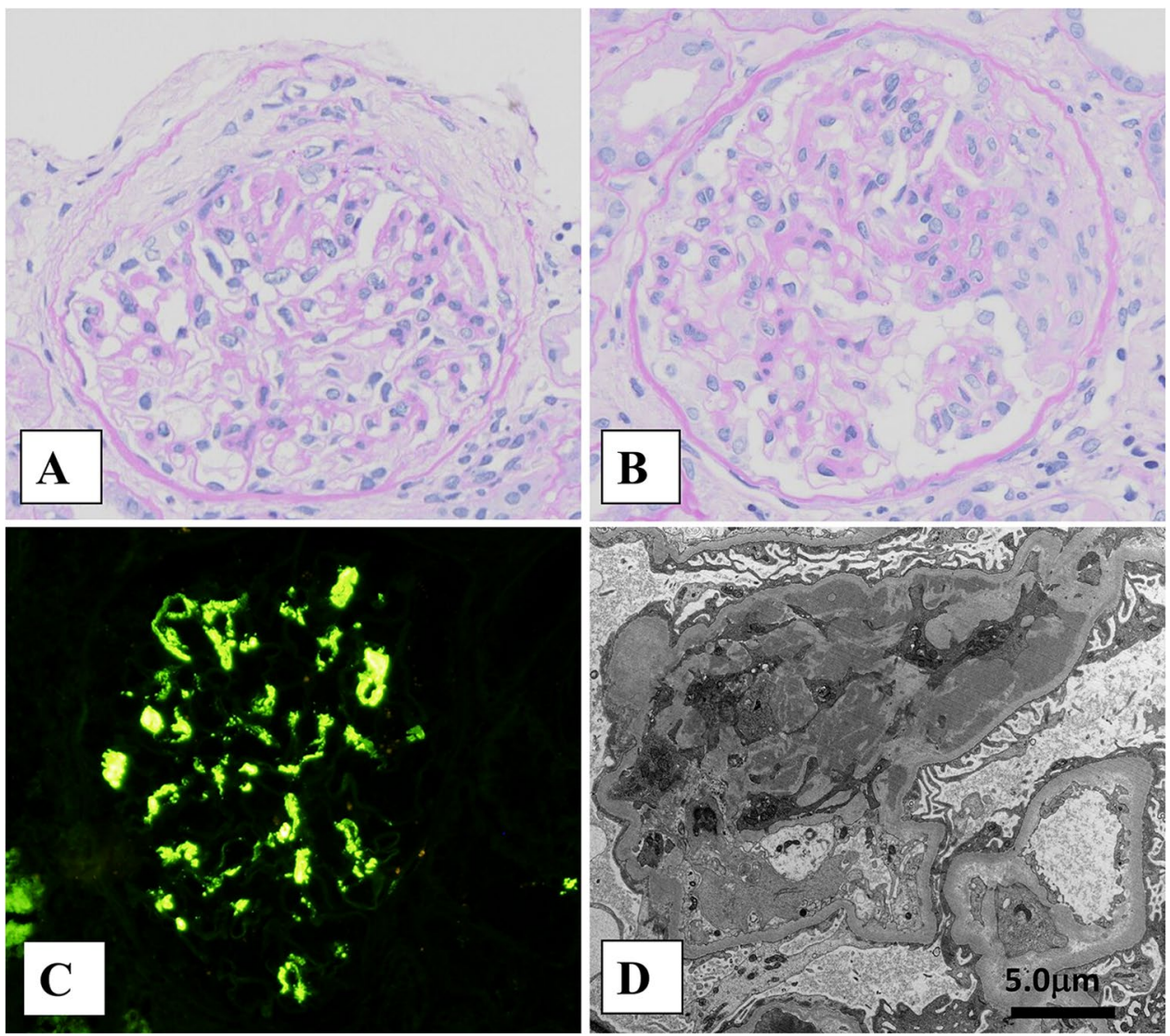

It is known that parenteral influenza vaccines that do not activate mucosal immune responses increase the IgA level [11]. A study examining the subclasses of IgA antibodies to intramuscularly injected influenza vaccine reported that patients with IgAN had a stronger monomeric response to subclass $\operatorname{IgA} 1$ than healthy subjects.

The markedly decreased platelet count in this case occurred simultaneously with gross hematuria. The various tests to diagnose thrombocytopenia, especially TMA, including TTP, hemolytic uremic syndrome, and other secondary TMA, were negative. There was no thrombus formation in the intracranial vessels, and the results of the antiplatelet antibodies and platelet factor 4 were negative, suggesting that thrombocytopenia associated with SARS-CoV-2 vaccine-related thrombus formation [7] was unlikely. Since this was not reported with the Pfizer vaccine, it is consistent. Thus, the causal relationship between the SARS-CoV-2 vaccine and thrombocytopenia was unclear even though the patient recovered spontaneously. In terms of treatment for thrombocytopenia, the effect of PE was also unclear in the present case.

In conclusion, the SARS-CoV-2 vaccines may cause exacerbation of both known and occult kidney diseases such as IgAN. However, this does not mean that vaccination should be avoided in those with known or occult kidney diseases but rather that more attention should be paid to patients who develop/exacerbate kidney diseases after receiving the SARS-CoV-2 vaccine regardless of the presence or absence of a definitive diagnosis. 


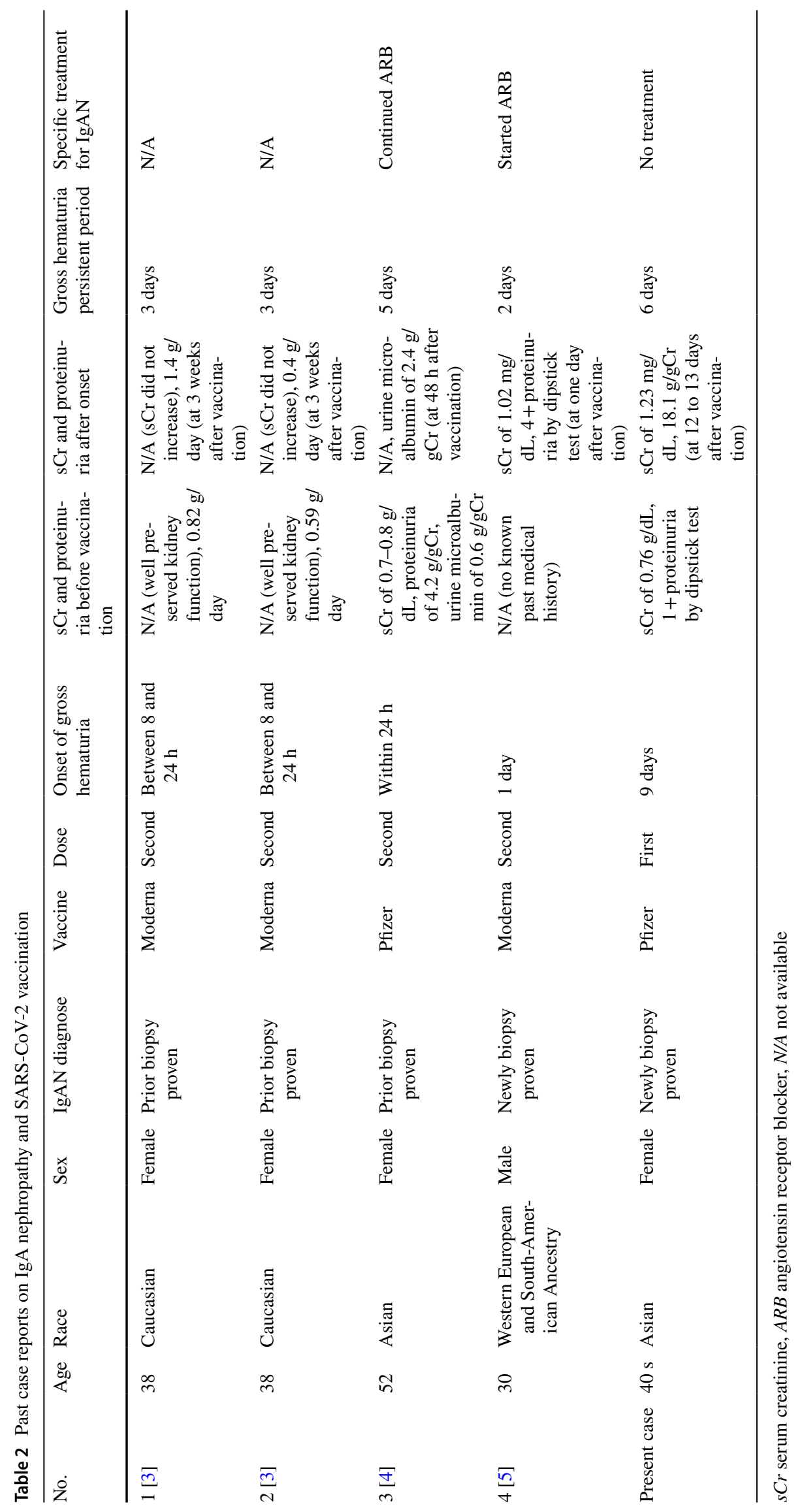


Author contributions YF and MY participated in the writing of the paper. YO, KY, ID, YS, and MY participated in the approval of final manuscript.

Funding This study has not been supported by any grant.

Availability of data and materials Corresponding author (MY) proves and administrates the patient's data transparency.

\section{Declarations}

Conflict of interest The authors have declared that no conflict of interest exists.

Ethical approval An ethical approval statement was not required for this manuscript since no specific information such as patient's age and admission or discharge date were included.

Informed consent The patient signed an informed consent to publish this report.

\section{References}

1. D'Marco L, Puchades MJ, Romero-Parra M, Gimenez-Civera E, Soler MJ, Ortiz A, et al. Coronavirus disease 2019 in chronic kidney disease. Clin Kidney J. 2020;13:297-306.

2. Morlidge C, El-Kateb S, Jeevaratnam P, Thompson B. Relapse of minimal change disease following the AstraZeneca COVID19 vaccine. Kidney Int. 2021;100:459.

3. Negrea L, Rovin BH. Gross hematuria following vaccination for severe acute respiratory syndrome coronavirus 2 in 2 patients with IgA nephropathy. Kidney Int. 2021;99:1487.
4. Rahim SEG, Lin JT, Wang JC. A case of gross hematuria and IgA nephropathy flare-up following SARS-CoV-2 vaccination. Kidney Int. 2021;1001:238.

5. Abramson M, Mon-Wei YuS, Campbell KN, Chung M, Salem F. IgA nephropathy after SARS-CoV-2 vaccination. Kidney Med. 2021. https://doi.org/10.1016/j.xkme.2021.05.002.

6. Donadio JV, Grande JP. IgA nephropathy. N Engl J Med. 2002;347:738-48.

7. Schultz NH, Sorvoll IH, Michelsen AE, Munthe LA, LundJohansen F, Ahlen MT, et al. Thrombosis and thrombocytopenia after ChAdOx1 nCoV-19 Vaccination. N Engl J Med. 2021;384:2124-30.

8. Trimarchi H, Barratt J, Cattran DC, Cook HT, Coppo R, Haas M, et al. Oxford Classification of IgA nephropathy 2016: an update from the IgA Nephropathy Classification Working Group. Kidney Int. 2017;91:1014-21.

9. Vojdani A, Kharrazian D. Potential antigenic cross-reactivity between SARS-CoV-2 and human tissue with a possible link to an increase in autoimmune diseases. Clin Immunol. 2020;217:108480.

10. Wisnewski AV, Campillo Luna J, Redlich CA. Human IgG and IgA responses to COVID-19 mRNA vaccines. PLoS ONE. 2021;16:e0249499.

11. Endoh M, Suga T, Miura M, Tomino Y, Nomoto Y, Sakai H. In vivo alteration of antibody production in patients with IgA nephropathy. Clin Exp Immunol. 1984;57:564-70.

Publisher's Note Springer Nature remains neutral with regard to jurisdictional claims in published maps and institutional affiliations. 